\title{
Weak fiber Bragg grating cascade sensor interrogation using microwave photonic filtering techniques
}

\author{
Javier Hervás Peralta ${ }^{1,{ }^{\star}}$, Amelia Lavinia Ricchiuti ${ }^{1}$, David Barrera ${ }^{1}$, Salvador Sales ${ }^{1}$, Luc Thévenaz ${ }^{2}$, \\ and José Capmany ${ }^{1}$ \\ ${ }^{1}$ ITEAM Research Institute, Universidad Politécnica de Valencia, Camino de Vera s/n, 46022 Valencia, Spain \\ ${ }^{2}$ Ecole Polytechnique Fédérale de Lausanne, Institute of Electrical Engineering, SCI-STI-LT Station 11, 1015 \\ Lausanne, Switzerland \\ *jaherpe2@teleco.upv.es
}

\begin{abstract}
A microwave photonic technique is presented to interrogate a $5 \mathrm{~m}$-long fiber, containing $9 \mathrm{~mm}$-long weak FBGs having $10 \mathrm{~mm}$ grating separation, used as a distributed sensor. Hot-spots as small as $20 \mathrm{~cm}$ have been measured with an accuracy of $0.5 \mathrm{~cm}$

OCIS codes: (060.3735) Fiber Bragg gratings; (280.0280) Remote sensing and sensors;
\end{abstract}

\section{Introduction}

Fiber Bragg Gratings (FBGs) play nowadays a key role in fiber optic systems and their applications, due to their benefits such as low complexity, polarization independence, low insertion loss, low cost and easy integration with optical fibers. In addition, since the material of which they are manufactured is a dielectric, FBGs are immune to electromagnetic interference (EMI), chemically non-active and spark free [1]. In the sensing domain [2], many methods have been proposed to interrogate such device with a view of implementing a temperature and/or strain sensor. In previous works, long high reflectivity FBGs have been used to detect hot spots [3]. Also, a temperature sensor based on a very long continuous weak FBG has been presented [4]. In this paper, a 5 m-long fiber containing $9 \mathrm{~mm}$-long weak FBGs in tandem featuring a $10 \mathrm{~mm}$ grating separation is used as sensing device. The interrogation method, based on microwave photonic (MWP) filtering technique and the novel design of the sensing device, let us get rid of some of the drawbacks presented in previous configurations allowing to detect and measure spot events along bigger distances (several tens of meters).

\section{Principle of operation}

The setup used to interrogate the $5 \mathrm{~m}$ fiber containing 500 weak FBGs written in tandem is depicted in Fig.1. The technique is based on the $S_{21}$ parameter measurement, which gives the electrical frequency response of the radiofrequency (RF) filter formed by the 500 hundred delayed taps of the original RF signal created by each FBG [5]. The output power of a broadband source (BBS), implemented with a SuperLED, was first filtered and then modulated by the MW signal coming from the Vector Network Analyzer (VNA) at the electro-optical modulator (EOM). The reason behind using an optically filtered broadband source, relies on the fact that an incoherent operation regime is desirable in terms of improved measurement stability [5]. Then, the modulated optical signal was launched into the device under test (DUT) and reflected taps of the input signal were generated [6]. Working in an incoherent regime limits the minimum delay between taps, because it must be larger than the source coherence time [3].

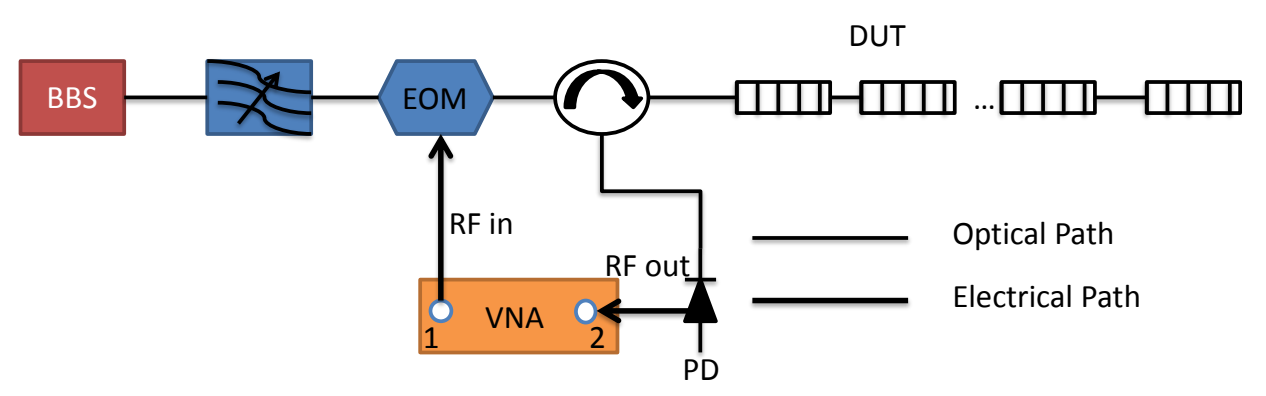

Fig. 1. Schematic diagram used to interrogate the $5 \mathrm{~m}$ fiber using MWP techniques 
The microwave signal was acquired after detection of these taps by the photodetector (PD) and the electrical frequency response is given by [5]:

$$
H(\omega)=\sum_{k=0}^{N} a_{k} e^{-i k \omega T_{k}}
$$

where $\omega$ is the microwave frequency, $a_{k}$ is the weight of each tap and $T_{k}$ is the delay of each tap. When $T_{k}=T$, this type of device has a periodic response, whose period is known as free spectral range (FSR), that is inversely proportional to the relative delay between samples [3]. It is well known that, this kind of MWP filter has $N-1$ minima between two consecutive maxima (i.e one FSR span), being $N$ the number of taps. Thus, the distance between minima in the electrical frequency response can be used to calculate the number of taps that contributed to the filter response. As the distance between consecutive FBGs is known, the length of the DUT can be calculated as well.

When a temperature shift is present in such device, the heated surface suffers a period shift and therefore a Bragg wavelength shift appear in the closest FBGs. If the optical filter is used to select the part of the source spectrum reflected by the heated FBGs, the distance between minima in $H(\omega)$ gives the DUT length that is subject to this temperature shift, allowing to use this MWP filter as a temperature sensor. Moreover, if a reference tap is located after the DUT, the position of this heated surface within the entire fiber can be also evaluated. In both cases, the inverse Fourier transform (IFT) of the electrical response gives the same results but with less accuracy in terms of length and more accuracy in terms of position. This occurs because many periods of the periodic event (periodic lobes in the electrical frequency response in this case) must be present to get a good accuracy using the IFT. Then, we can identify the hot-spot events, their length and location in fibers containing weak FBGs. Also, the temperature shift could be measured using an Optical Spectrum Analyzer if the thermal coefficient of the FBGs is known.

\section{Experimental measurements and results}

Due to limitations in the bandwidth of the equipment used and, since the objective was to design a low cost interrogation system sensor, the electrical response has been limited only up to $500 \mathrm{MHz}$, letting us to measure hot spots larger than $20 \mathrm{~cm}$. A $26 \mathrm{~cm}$ long section of the DUT was heated and the band reflected by this section was selected by the optical filter to prove this system can detect this hot spot. The spectrum of this heated section is shown in Fig.2(a). This spectrum has a minimum at $0.3883 \mathrm{GHz}$, equivalent to a heated surface of $26.53 \mathrm{~cm}$, giving a resolution of $0.5 \mathrm{~cm}$. Then, in order to prove that also the position of the spot event along the DUT could be evaluated, a $79 \mathrm{~cm}$ section located around the centre of the fiber was heated and a reference tap (fiber end with physical contact (PC) connector) was located $7 \mathrm{~m}$ after the DUT.

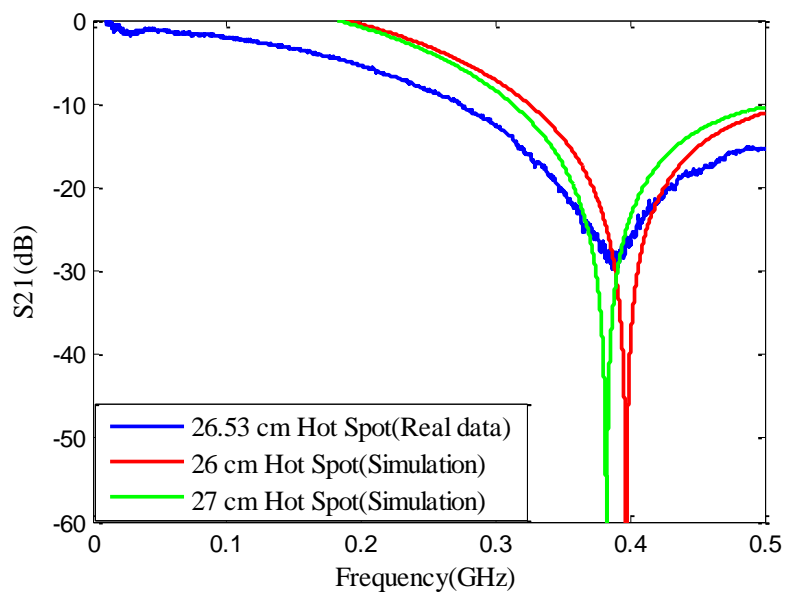

a)

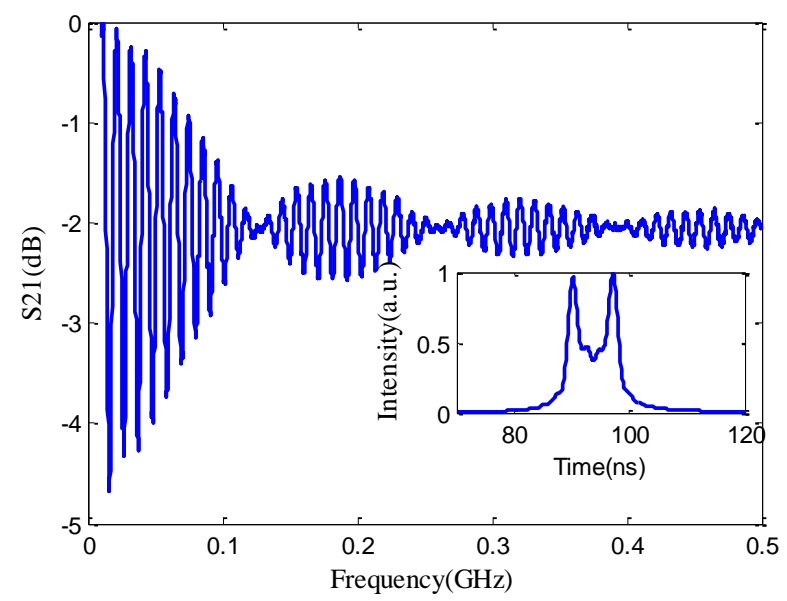

b)

Fig. 2. a) Electrical frequency response of a $26 \mathrm{~cm}$ hot spot (Real data and simulations). b) Electrical frequency response of a $79 \mathrm{~cm}$ hot spot centred at $2.67 \mathrm{~m}$ of the fiber end. Inset: IFT of the previous electrical frequency response. 
The results of this experiment are shown in Fig.2(b). Two modulations can be seen in the electrical response, the first one due to the filter generated by the $79 \mathrm{~cm}$ of the fiber and the other one due to the relation between the filter and the reference tap. Although the length of the heated surface was calculated with the electrical response, its position within the DUT was calculated with the IFT, thanks to its larger accuracy, since as the experiment has been performed up to $500 \mathrm{MHz}$, only a few lobes have been originated due to the spot event length, but the lobes caused by the reference tap have a smaller period in term of frequency and their effect is very present at the IFT. The inset of Fig.2(b) shows two peaks located at 97.24 and $90.2 \mathrm{~ns}$, whose centre is located at $93.72 \mathrm{~ns}$, which means that the heated area is centred at $2.67 \mathrm{~m}$ in relation to the $5 \mathrm{~m}$ fiber end.

\section{Conclusions}

In this work, we have proposed and experimentally demonstrated a microwave photonic filtering technique to interrogate a photonic sensor based on a distributed weak fiber Bragg gratings MW filter. We have demonstrated that we can identify the hot-spot events, their length and location in fibers containing weak FBGs. The measurement setup is based on the MWP filter principle operation, which consist on measure the $S_{21}$ parameter which gives the electrical frequency response of the MW filter. In this technique, a broadband source is filtered to ensure the incoherence between taps and also to select a portion of the broadband spectrum, either the portion reflected by the original filter or the portion reflected by the hot spot. The results given by this technique have an accuracy of $0.5 \mathrm{~cm}$ and we were able to measure hot spots as small as $20 \mathrm{~cm}$. To measure smaller hot spots the photodetector and modulator bandwidths must be broader, but this setup could be used without changes in many applications due to its accuracy, low complexity and the absence of broadband components. It can be mentioned that the same principle of operation could be used to perform a strain sensor, since this kind of event would create a similar Bragg wavelength shift and that the same results and accuracy would be possible if a longer fiber $(50 \mathrm{~m}$, for instance) had been used, giving the opportunity of having a very large sensed area.

We thank Johan Viekken from FBGS International to let us the $5 \mathrm{~m}$-long fiber containing $9 \mathrm{~mm}$-long weak FBGs. The authors also acknowledge the Infraestructura FEDER UPVOV08-3E-008, FEDER UPVOV10-3E-492, the Spanish MCINN through the project TEC2011-29120-C05-05, the Valencia Government through the Ayuda Complementaria ACOMP/2013/146, the financial support given by the Research Excellency Award Program GVA PROMETEO 2013/012.

\section{References}

[1] B. Culshaw, "Optical fiber sensor technologies: opportunities and perhaps pitfalls," J. Lightwave Technol. 22, 39-50 (2004).

[2] A. D. Kersey, M. A. Davis, H. J. Patrick, M. LeBlanc, K. P. Koo, C. G. Askins, M. A. Putnam, and E. J. Friebele, "Fiber grating sensors," J. Lightwave Technol. 15, 1442-1463 (1997).

[4] A.L Ricchiuti, D. Barrera, S. Sales, L. Thévenaz, and J. Capmany, "Long fiber Bragg grating sensor interrogation using discrete-time microwave photonic filtering techniques," Opt. Express 21, 28175-28181 (2013).

[5] L. Thévenaz, S. Chin, J. Sancho, S. Sales," Novel_technique for distributed fibre sensing based on faint long gratings (FLOGs)," OFS2014 23rd International Conference on Optical Fiber Sensors, Santander, 2014.

[5] J. Capmany, J. Mora, I. Gasulla, J. Sancho, J. Lloret, and S. Sales, "Microwave Photonic Signal Processing," J. Lightwave Technol. 31, 571$586(2013)$

[6] L. R. Chen, S. D. Benjamin, P. W. E. Smith, and J. E. Sipe, "Ultrashort pulse reflection from fiber gratings: a numerical investigation," J. Lightwave Technol. 15, 1503-1512 (1997). 